\title{
Aplicação de programação por metas binária - mista em uma empresa do setor sucroenergético
}

\section{Application of mixed binary goal programming in an enterprise in the sugar and energy sector}

\author{
Aneirson Francisco da Silva ${ }^{1}$ \\ Fernando Augusto Silva Marins ${ }^{1}$ \\ José Arnaldo Barra Montevechi²
}

\begin{abstract}
Resumo: A Programação por Metas, ou Goal Programming (GP), é uma importante abordagem analítica que tem sido aplicada com sucesso na solução de importantes problemas de decisão. Propõe-se um modelo de Programação por Metas Binária-Mista, ou Mixed-Binary Goal Programming (MBGP), para otimizar o planejamento agregado da produção e da distribuição de uma empresa do setor sucroenergético, que incorpora a cogeração de energia. Deste modo, o modelo aborda os processos de produção de açúcar, de álcool, de melaço e derivados e da cogeração de energia. Contempla, também, as etapas agrícolas, industriais e de distribuição, possibilitando que as decisões sejam tomadas em um horizonte de planejamento semanal, incluindo a safra e entressafra. A aplicação deste modelo em uma usina de açúcar e álcool brasileira auxiliou na determinação de políticas ótimas de produção e distribuição, gerando resultados financeiros e operacionais interessantes para a empresa, além de gerar relatórios de exportação de energia aderentes à realidade da usina estudada.
\end{abstract}

Palavras-chave: Programação por metas. Programação de metas binária-mista. Planejamento agregado. Cogeração de energia. Usinas de açúcar e álcool.

Abstract: The Goal Programming (GP) is an important analytical approach that has been successfully applied in relevant real-world decision making problems. This paper proposes a Mixed-Binary Goal Programming (MBGP) model to optimize production and distribution planning of a energy cogeneration sugar and ethanol milling company. Therefore, the MBGP model integrates the production process of sugar, alcohol, molasses, and energy cogeneration, and it enables decision-making in the agricultural, industrial, and distribution phases on a weekly-basis planning horizon including the harvest season and the periods between harvests. The model was applied in a Brazilian sugar and ethanol milling company, and it contributed to the formulation of production and distribution optimum policies yielding good financial and operational results in addition to raising energy export in conformity with the reality of the plant.

Keywords: Goal programming. Mixed binary goal programming. Aggregate production and distribution planning. energy cogeneration. Sugar and ethanol milling plants.

\section{Introdução}

A agroindústria brasileira da cana tem enfrentado uma grande mudança organizacional. Aspectos de gestão nesta indústria estão mudando, devido à importância de seus produtos, especialmente o etanol e a eletricidade (PAIVA; MORABITO, 2009, 2011; PAIVA, 2009).

Com respeito às pesquisas nesta área envolvendo pesquisadores de outros países, podem ser descritas algumas importantes contribuições. Mathew e Rajendran (1993) utilizaram a simulação a eventos discretos, com o objetivo de avaliar a programação de manutenções de uma usina, visando estabelecer intervalos razoáveis entre essas paradas. Kawamura, Ronconi e Yoshizaki (2006) utilizaram a Programação Linear Multiperíodo para auxiliar nas decisões relacionadas a transporte de produtos e armazenagens em uma cooperativa de produtores de açúcar e álcool. Por sua vez, Cock, Luna e Palma (2000) desenvolveram uma metodologia para escolha de variedades de cana por meio da análise de custo total de processamento dessas variedades. Por meio da simulação, Higgins e Davies (2005) realizaram um estudo que visava à

\footnotetext{
Faculdade de Engenharia, Universidade Estadual Paulista - UNESP, Av. Ariberto Pereira da Cunha, 333, CEP 12516-410, Guaratinguetá, SP, Brasil, e-mail: aneirson@yahoo.com.br, fmarins@ feg.unesp.br

2 Instituto de Engenharia de Produção e Gestão - IEPG, Universidade Federal de Itajubá - UNIFEI, Av. Benedito Pereira dos Santos, 1303, Itajubá, MG, Brasil, e-mail: montevechi@unifei.edu.br
} 
melhoria do planejamento logístico de transporte de cana. Já Higgins et al. (2004), Higgins (2006), Milan, Fernandez e Aragones (2006), Grunow, Günter e Westinner (2007) aplicaram modelos monobjetivos em problemas sucroalcooleiros.

Paiva (2009) comenta que, no Brasil, a partir de 1990, com a abertura do mercado de açúcar e álcool e os avanços na tecnologia de microinformática e computação, houve o surgimento de várias contribuições com a utilização de métodos quantitativos na indústria sucroalcooleira. Iannoni e Morabito (2006) investigaram um sistema de recepção de cana de açúcar de uma usina, por meio da simulação a eventos discretos, cujo objetivo foi avaliar a logística de transporte.

Paiva e Morabito (2009) desenvolveram um modelo monobjetivo que integrava as etapas agrícolas e industriais, auxiliando nas decisões de safra. Paiva e Morabito (2009) aplicaram modelos monobjetivos em problemas sucroalcooleiros.

Paiva (2009) e Paiva e Morabito (2011) aplicaram métodos de otimização sob incerteza, conhecidos por: Otimização Estocástica Robusta e Otimização Robusta - em problemas reais de usinas cooperadas do setor sucroenergético.

Ainda no Brasil, alguns trabalhos recentes podem ser citados. Silva (2009) aplicou um modelo multiobjetivo da Programação por Metas, ou Goal Programming (GP), em problemas reais sucroalcooleiros. Este modelo integrou as etapas agrícolas e industriais, visando auxiliar nas decisões na safra e entressafra. Já Silva et al. (2009) desenvolveram um modelo multiobjetivo combinado com o Analytic Hierarchy Process (SAATY; SHANG, 2007), cujo objetivo foi de propiciar maior interação dos gestores com o modelo matemático, além de potencializar a flexibilidade do modelo proposto. Silva et al. (2010) desenvolveram um modelo multiobjetivo sob incerteza, conhecido por otimização Multiobjetivo Fuzzy, cujo objetivo foi incorporar as incertezas presentes nos processos sucroenergéticos.

O objetivo geral desta pesquisa foi desenvolver um modelo de Programação por Metas Binária-Mista para auxiliar nas decisões de planejamento da colheita, planejamento agregado da produção e na cogeração de energia de uma usina de açúcar e álcool. A seguir, estão algumas das características do trabalho:

- Trata a integração das etapas agrícola, industrial e logística em um único modelo MBGP para auxiliar nas decisões de safra e entressafra;

- Modela o processo de cogeração de energia elétrica considerando desde a necessidade de vapor para os processos de moagem, destilação de álcool e fabricação do açúcar até a exportação e comercialização da energia excedente;
- Mostra a aplicação do modelo MBGP em problemas reais de grande porte; $\mathrm{e}$

- Permite avaliar a eficiência dos solvers CONOPT, DICOPT e CPLEX (GENERAL..., 2010a, b, c) que foram utilizados em conjunto, bem como a qualidade da solução ótima gerada; e

- Facilita a geração de cenários e análise de sensibilidade do modelo.

Este artigo está organizado em seções. Na seção 2, é feita uma breve fundamentação sobre a GP e o modelo MBGP. A seção 3 apresenta o método utilizado na pesquisa. A seção 4 refere-se ao desenvolvimento e otimização do modelo; a seção 5 contempla a discussão dos resultados e o direcionamento para novas pesquisas, sendo seguida pelas referências bibliográficas.

\section{Programação por metas e programação por metas binária-mista}

\subsection{Programação por metas}

Ignizio (1976) argumentou que problemas de otimização não devem apenas procurar uma solução ótima, mas gerar soluções que permitam o aprendizado sobre a situação abordada. Assim, a Programação por Metas (Goal Programming - GP), que inclui modelos de Programação Multiobjetivo (PMO), segundo Deb (2001) e Chang (2007), difere consideravelmente da forma clássica de otimização adotada em outras áreas da Programação Matemática, conforme afirmam Martel e Aouni (1998), Min e Storbeck (1991), Charnes e Cooper (1961), Ignizio (1976) e Charnes et al. (1979).

A abordagem da GP original considera que os gestores devem escolher um valor alvo para cada meta $i$ e decidir qual é a penalização mais adequada para as variáveis de desvio associadas à obtenção de valores acima $\left(d_{i}^{+}\right)$ou abaixo $\left(d_{i}^{-}\right)$deste valor alvo.

Há ainda informações importantes sobre estudos e aplicações gerais da GP em Charnes e Cooper (1961), Ignizio (1976) e Caballero, Gómez e Ruiz (2009). Outros trabalhos interessantes, aplicando variações de modelos da GP, em áreas variadas, estão relacionados a seguir:

- Gokcen e Erel (1997) aplicaram um modelo de GP em uma linha de montagem;

- Leung e Chan (2009) estudaram a aplicação de um modelo de GP no planejamento agregado da produção com restrição de recurso;

- Liao (2009) desenvolveu um novo modelo de GP conhecido por Multi-segment Goal Programming (MSGP), visando incorporar a incerteza na estimação dos coeficientes tecnológicos (Left-hand side); 
- Chang $(2007,2008,2010)$ desenvolveu novos modelos de GP, conhecidos por Multi-choice Goal Programming (MCGP) e por Revised Multi-Choice GP (RMC-GP), que incorporam às incertezas presentes na estimação das limitações ou recursos (Right-hand side);

- Ustun (2012) propôs uma nova formulação da MCGP com base numa função cônica escalar, e segundo este autor, a nova formulação se mostrou mais eficientes, e com grandes vantagens em relação aos modelos MCGP e RMC-GP; e

- Yaghoobi e Tamiz (2007) desenvolveram um modelo Fuzzy Goal Programming para tratar incertezas presentes nas limitações (Right hand side).

Existem diversas variantes de modelos da GP e, de acordo com Yaghoobi e Tamiz (2007) e Romero (2004), os três modelos principais de GP, que são utilizados com maior frequência são: o WGP, o LGP e o MAGP. Exemplos de aplicações destes modelos podem ser encontrados em várias publicações (TAMIZ; JONES; EL-DARZI, 1995; TAMIZ; JONES; ROMERO, 1998; JONES; TAMIZ, 2002; AOUNI \& KETTANI, 2001; JONES; BARNES, 2002; ROMERO, 2001, 2004; AOUNI; MARTEL; HASSAINE, 2009; CABALLERO; GÓMEZ; RUIZ, 2009; CHANG, 2004; ÖZCAN; TOKLU, 2009).

Para Martel e Aouni (1998), o modelo de WGP tem como perspectiva atender aos objetivos e metas estabelecidas pelos gestores, por meio da atribuição de pesos associados a cada variável de desvio e, desta forma, estabelecer uma ordem de prioridade entre os diversos objetivos. A formulação do modelo original WGP, que foi criado por Charnes e Cooper (1961), pode ser expressa pelo programa (1) - (4):

$$
\begin{gathered}
\operatorname{Min} \mathrm{Z}= \\
\text { s.a: } \sum_{j=1}^{n} a_{i j} x_{j}-d_{i}^{+}+d_{i}^{-}=g_{i} \\
A x \leq c \\
x_{j}, d_{i}^{+}, \text {e } d_{i}^{-} \geq 0 \text { para } \\
i=1,2, \ldots, p, \text { e } j=1,2, \ldots, n,
\end{gathered}
$$

sendo $f_{i}(x)$ as múltiplas funções objetivo, $W_{i}$ representa o peso associado a cada variável de desvio $d_{i}^{+}, d_{i}^{-}$, que são associadas à realização acima ou abaixo do valor alvo da meta $g_{i}$. Assim, (2) representa as restrições flexíveis do modelo e (3) representa as restrições rígidas do modelo, ou seja, aquelas que devem ser necessariamente satisfeitas.

Caballero, Gómez e Ruiz (2009) desenvolveram uma pesquisa tipo survey sobre a GP, investigando problemas e tipos de variáveis, aos quais se aplicam a GP. Estes autores verificaram que há mais aplicações dos modelos WGP e MAGP do que de outros modelos da GP, e que eles são utilizados com maior frequência em problemas industriais, depois em áreas que tratam com recursos naturais e finalmente na área de negócios. Eles também investigaram as características dos problemas reais abordados pelos modelos de GP e concluíram haver ocorrência de variáveis Fuzzy, binárias e inteiras, que geram maior dificuldade de resolução dos modelos, daí a necessidade de se disponibilizar modelos adequados e confiáveis, e métodos de solução que sejam factíveis de serem implementados pelos gestores destas empresas, proporcionando-lhes, em tempo hábil, elementos para a tomada de decisão.

Outro ponto que merece destaque é a carência da aplicação de modelos de GP em problemas reais do setor sucroenergético brasileiro, que tem uma importância estratégica relevante para o País. Em outras palavras, no Brasil tem-se explorado pouco a aplicação de modelos de GP na otimização de problemas nesta área.

Destacam-se, neste contexto, as contribuições de Munhoz e Morabito (2001, 2010), Cunha e Caixeta Filho (2002), Silva et al. (2006, 2010, 2012) e Silva (2009), que têm características diferentes daquelas do modelo aqui aplicado, conforme já comentado.

Todavia, pode ocorrer, em alguns problemas, que os gestores não sejam capazes de atingir alvos específicos, ou não estão interessados nisso. De fato, podem existir situações, em que há Objetivos (e Metas associadas) conflitantes, ou seja, há relações de trade-offs entre eles, e o analista gostaria de poder decidir na escolha de quais Metas (Goals) seriam atingidas e quais não. Os métodos tradicionais de GP têm dificuldade para tratar tais situações (CHANG, 2004). Neste cenário, modelos da Programação por Metas Binária-Mista (MBGP) podem ser utilizados, visando estabelecer quais metas deverão ser atingidas plenamente.

\subsection{Programação por Metas Binária-Mista}

O modelo MBGP geral pode ser descrito pelo programa (5) - (9), conforme Chang (2000):

$$
\begin{gathered}
\operatorname{MinZ}=\sum_{i=1}^{n}\left(d_{i}^{+}+d_{i}^{-}\right) y_{i} \\
\text { s.a: }\left(f_{i}(X)-g_{i}\right) y_{i}=d_{i}^{+}-d_{i}^{-}, \quad i=1,2, \ldots, n,(6) \\
d_{i}^{+}, d_{i}^{-} \geq 0, \quad i=1,2, \ldots, n \\
y_{i} \in R_{i}, \quad i=1,2, \ldots, n
\end{gathered}
$$


$X \in F$ ( $F$ é um conjunto de soluções viáveis) (9) sendo $y_{i}$ a variável (binária) de controle para $i$-ésima meta $i$, o comportamento de $y_{i}(0$ ou 1$)$ depende de $R_{i}$, que é a restrição ambiental da $i$-ésima meta $i$, e as demais variáveis são análogas àquelas do modelo WGP.

Como o modelo MBGP geral é não linear, Chang (2000) propôs linearizá-lo, conforme o programa $(10)-(21)$ :

$$
\begin{gathered}
\operatorname{MinZ}=\sum_{i=1}^{n} z_{i}^{+}+z_{i}^{-} \\
\text {s.a: } h_{i}(X)-k_{i}=d_{i}^{+}-d_{i}^{-}, \quad i=1,2, \ldots, n,, \\
d_{i}^{+}+\left(y_{i}-1\right) M \leq z_{i}^{+} \leq \\
\left(1-y_{i}\right) M+d_{i}^{+}, \quad i=1,2, \ldots, n, \\
z_{i}^{+} \leq y_{i} M, \quad i=1,2, \ldots, n, \\
d_{i}^{-}+\left(y_{i}-1\right) M \leq z_{i}^{-} \leq \\
\left(1-y_{i}\right) M+d_{i}^{-}, \quad i=1,2, \ldots, n, \\
z_{i}^{-} \leq y_{i} M, \quad i=1,2, \ldots, n, \\
f_{i}(X)+\left(y_{i}-1\right) M \leq h_{i}(X) \leq \\
\left(1-y_{i}\right) M+f_{i}(X), \quad i=1,2, \ldots, n, \\
h_{i}(X) \leq y_{i} M, \quad i=1,2, \ldots, n, \\
k_{i}=g_{i} y_{i}, \quad i=1,2, \ldots, n, \\
y_{i} \in R_{i} \quad i=1,2, \ldots, n,
\end{gathered}
$$

$X \in F$ ( $F$ é um conjunto de soluções viáveis) (20)

$$
d_{i}^{+}, d_{i}^{-} \geq 0, \quad i=1,2, \ldots, n,
$$

sendo $M$ um valor suficientemente grande (big $M$ ) e as demais variáveis são análogas aos modelos anteriores.

Aqui, o modelo MBGP será adotado para modelar a produção agregada de uma usina típica do setor sucroenergético com a incorporação da cogeração de energia elétrica, por meio da queima do bagaço de cana.

Destaque-se que o modelo MBGP é aplicável em problemas monoestágio, multiproduto, multiprocesso, dinâmico, capacitado e combina decisões de dimensionamento e sequenciamento de lotes (FERREIRA; MORABITO; RANGEL, 2009; ARAÚJO; ARENALES, 2000; ZIAEE; SADJADI, 2007).
A pressuposição principal deste tipo de modelo (MBGP) é que, em um período de análise, só poderá ser utilizado um lote, ou seja, tem-se um modelo de produção tipo "tudo ou nada" (ou small bucket), ou seja, só ocorre custo de preparação (setup) quando a produção de um novo lote for iniciada (PAIVA, 2009; PAIVA; MORABITO, 2009, 2011).

Seguem-se algumas justificativas que atestam a relevância da pesquisa realizada. Jamalnia e Soukhakian (2009) comentam que técnicas tradicionais de Programação Matemática não são adequadas para resolver problemas reais de planejamento agregado da produção, que apresentam número elevado de variáveis e de restrições, e com vários objetivos - que podem ser conflitantes - o que se reflete na dificuldade de modelagem e obtenção de soluções ótimas que sejam úteis para a tomada de decisão em tempo real.

Portanto uma contribuição científica deste trabalho é gerar novas e importantes aplicações da GP, particularmente da MBGP, em problemas reais em usinas sucroenergéticas.

\section{Material e métodos}

De acordo com Bertrand e Fransoo (2002) e Miguel et al. (2012), este trabalho pode ser classificado como sendo uma pesquisa aplicada, com objetivos empíricos descritivos, pois o modelo desenvolvido descreve relações causais que podem existir na realidade, favorecendo a compreensão de processos reais. A forma de abordar o problema é quantitativa, sendo o método de pesquisa a modelagem.

\subsection{Etapas da pesquisa}

As fases da pesquisa estão contempladas na Figura 1 e explicadas na sequência:

- Identificação do problema-Foram realizadas visitas em algumas usinas e identificados os processos tradicionalmente adotados e, após o mapeamento destes processos, houve o desenvolvimento do modelo MBGP, sendo utilizadas como base as pesquisas de Paiva $(2006$, 2009) e Paiva e Morabito, 2007, 2009, 2011;

- Coleta de dados (Seção 4) - Utilizaram-se relatórios internos de produção e de informação agrícola de uma usina escolhida como objeto do estudo;

- Modelagem (Seção 4) - A modelagem dos processos da usina escolhida contempla as decisões de safra e entressafra (52 semanas);

- Solução do modelo (Seção 5) - Adotou-se a linguagem de modelagem General Algebraic Modeling System (GAMS 23.6.5) e os Solvers CONOPT (GAMS, 2010a), DICOPT (GAMS, 2010b) e CPLEX 12.2.1 (GAMS, 2010c) que 


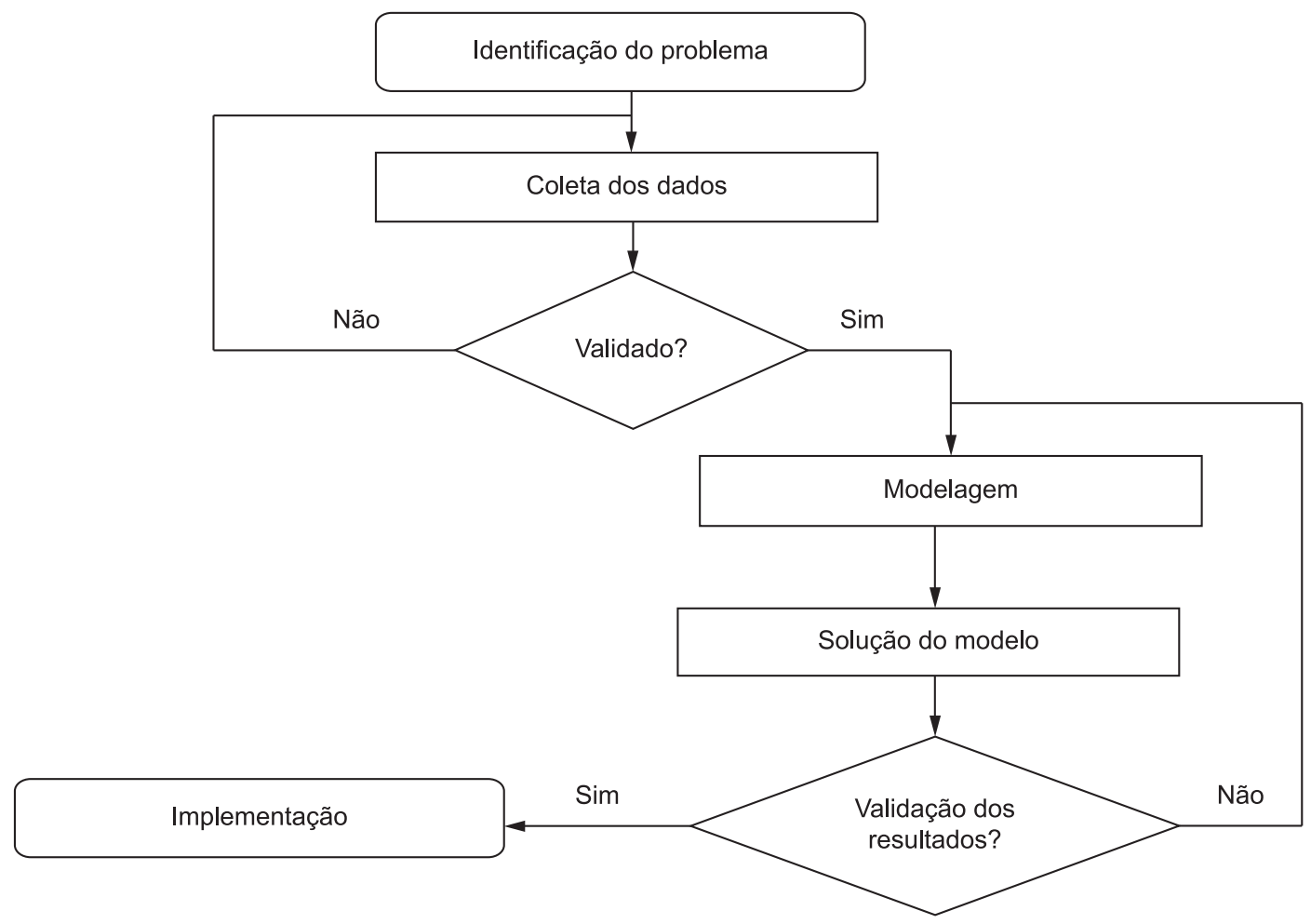

Figura 1. Etapas da pesquisa.

trabalharam de forma conjunta, atuando em um Modelo de Programação Não-linear Mista, ou Mixed Integer Nonlinear Programming - MINLP;

- Validação dos resultados (Seção 5) - foi feita com o apoio dos gestores da usina, que analisaram se os planos de produção gerados pelo modelo eram viáveis e, também, com a comparação de outros resultados do modelo com aquilo que vinha sendo praticado pela usina. Uma validação estatística da aderência do modelo à realidade da usina estudada foi feita por meio de um teste de hipótese não paramétrico; e

- Implementação - Esta etapa deve ser aprovada pelo Board da empresa estudada e foge do escopo deste trabalho.

\subsection{Descrição do problema}

A Figura 2 mostra o mapeamento dos processos de produção dos açúcares, álcoois, melaços e derivados, conforme exposto em Paiva (2009).

A usina escolhida, para a aplicação do modelo MBGP, situa-se na região sudeste do Brasil, tendo, anualmente, capacidade para esmagar 1,6 milhões de toneladas de cana, produzir 120.000 toneladas de açúcares e $95.000 \mathrm{~m}^{3}$ de álcool. Na safra de 2008/2009, a usina produzia o álcool AEHC e os açúcares VHP,
VVHP e Cristal, comercializava o bagaço e o óleo fúsel, e possuía duas opções de estocagem, sendo que a opção de estoque de terceiros (Eterc) era utilizada esporadicamente. Na fase de exportação, a usina utilizava dois portos para realizar a exportação (Santos e Rio Janeiro), empregando duas opções de frota (própria e terceirizada).

Os objetivos estabelecidos pelos gestores da usina foram: minimização do custo total da matéria prima, do custo total de estocagem, do custo total do transporte agrícola, do custo total de produção, do custo total de logística para exportação; a maximização da produção total de açúcares e etanol; e a maximização da venda de energia elétrica.

Os valores alvos das metas associadas aos objetivos foram estimados com base em dados históricos de relatórios contábeis e de produção e, também, a partir de sugestões da alta gerência da usina. As metas associadas a estes objetivos estão representadas pelo índice 5 no modelo desenvolvido na seção 4 .

\section{Modelagem do problema}

A seguir apresentam-se os índices, conjuntos, parâmetros, variáveis, inequações e equações utilizadas no modelo MBGP.

Conforme já citado, no modelo MBGP, estabelecido conforme as expressões de (22) - (56) fornecidas adiante, que foi aplicado em uma usina sucroenergética 


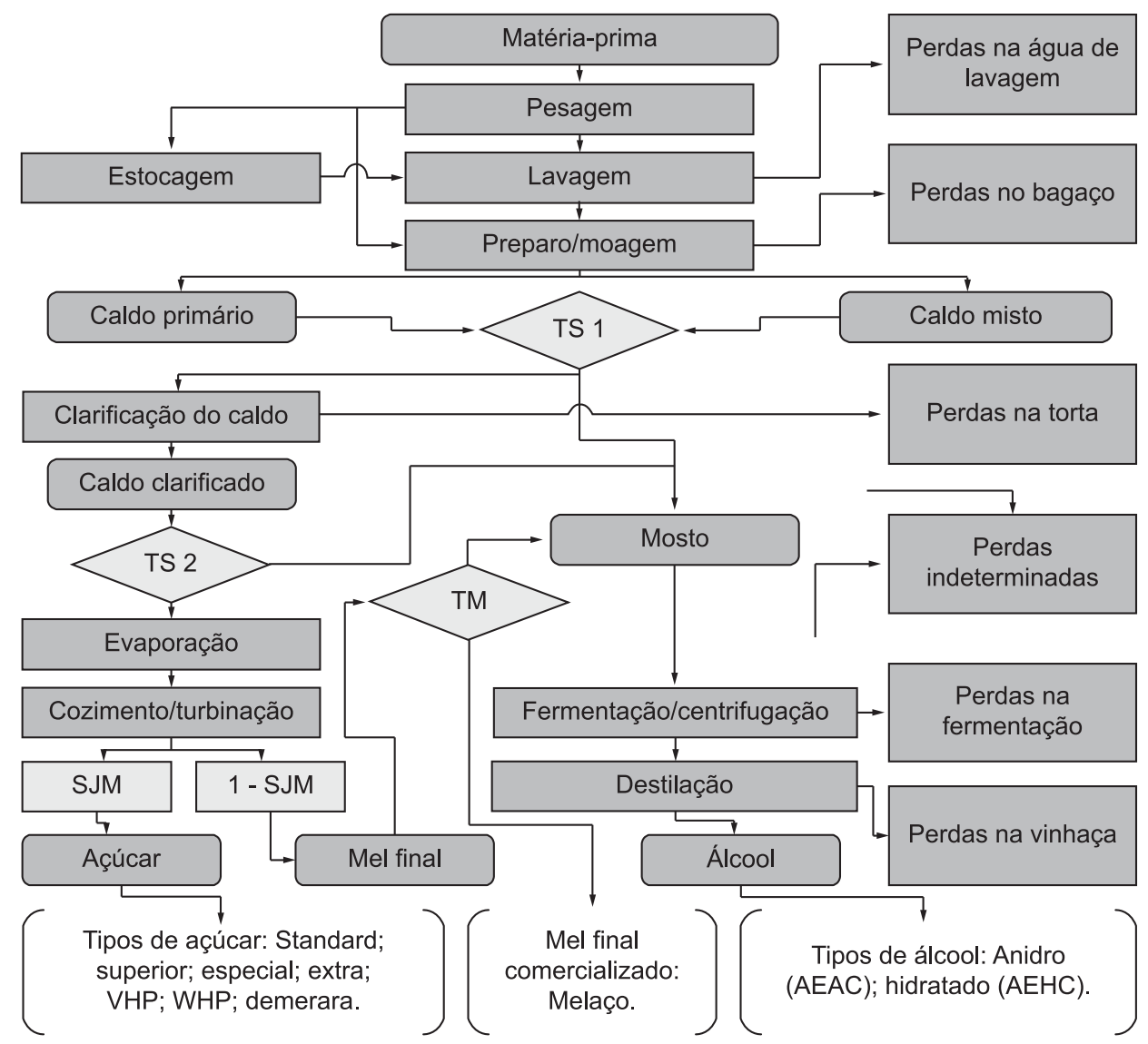

Figura 2. Fluxograma do processo de produção de açúcar, álcool e melaço. Fonte: (PAIVA, 2009).

escolhida como objeto do estudo, a seguir descrito, apresenta algumas mudanças em relação ao modelo do Paiva e Morabito (2007). As principais são: utilização de um modelo de Programação Multiobjetivo, desenvolvido por Chang (2000), para tratar de modelos binários mistos; a inclusão da cogeração de energia elétrica como produto de uma usina; e a integração das etapas agrícola, industrial e de logística em um único modelo multiobjetivo.

\section{Índices}

$p$ Produtos, $p \in P, P=\{$ VHP, VVHP, Cristal, AEHC $\}$;

$e$ Opção de estoque, $e \in E, E=\{$ Eprop, Eterc $\}$;

$t \quad$ Períodos, $t \in T, T=\{1,2, \ldots, 52\}$;

pp Coprodutos, $p p \in P P, P P=\{$ Bagaço e Óleo Fúsel $\}$;

$m$ Fonte de matéria prima, $m \in M, M=\{$ própria, fornecida\};

$f$ Opção de transporte agrícola, $f \in F, F=\{F p r o p$, Fterc \};

$i$ Destinos para exportação, $i \in I, I=\left\{\right.$ Dest $_{1}$, Dest $\left._{2}\right\}$;

$l$ Opção de transporte para distribuição, $l \in L, L=$ $\left\{T L_{1}, T L_{2}\right\}$;

$p_{1}$ Produção do açúcar VHP; $p_{2}$ Produção do açúcar VVHP;

$p_{3}$ Produção do açúcar Crystal;

$p_{4}$ Produção do AEHC;

$\theta$ Pertinente às metas, $\theta \in \Theta, \Theta=\{1,2, \ldots, 10\}$.

\section{Conjuntos}

$P$ Conjunto associado aos produtos, $P=\{V H P$, VVHP, Cristal, AEHC

$E$ Conjunto associado às opções de estoque, $E=\{$ Eprop, Eterc $\}$

$T$ Conjunto associado aos Períodos, $T=\{1,2, \ldots, 52\}$

$P P$ Coprodutos, $P P=\{$ Bagaço e Óleo Fúsel $\}$

$M$ Fonte de matéria-prima, $M=\{$ própria, fornecida $\}$

$F$ Opção de transporte agrícola, $F=\{$ Fprop, Fterc $\}$

$I$ Destinos para exportação, $I=\left\{\right.$ Dest $_{1}$, Dest $\left.{ }_{2}\right\}$

$L$ Opção de transporte para distribuição, $L=\left\{\mathrm{TL}_{1}, \mathrm{TL}_{2}\right\}$

$\Theta$ Pertinente às metas, $\Theta=\left\{\mathrm{Goal}_{1}, \mathrm{Goal}_{2}, \ldots, \mathrm{Goal}_{10}\right\}$.

\section{Parâmetros da Etapa Agrícola}

$M \min _{t} \quad$ Moagem mínima semanal [ton/sem]

$M \max _{\mathrm{t}}$ Moagem máxima semanal [ton/sem] 
$C T_{f} \quad$ Capacidade semanal do transporte agrícola $f$ [ton/semana]

$\phi \quad$ Tempo efetivo de operação da indústria durante um período $t$ [\%]

$\beta_{f t} \quad$ Disponibilidade da frota própria $f$ durante o período $t[\%]$

Disp $_{m 0} \quad$ Previsão de colheita de cana por fonte de matéria-prima $m$ [ton]

$C_{m t} \quad$ Matriz dos custos agrícolas. Representa o custo da matéria-prima $m$ no período $t$ [\$/ton]

$L_{f t} \quad$ Custo variável de corte, carregamento e transporte $f$ no período $t$ [\$/ton]

$M_{m 0}^{\prime} \quad$ Quantidade de cana colhida antes do primeiro período de programação dos fornecedores de cana $m$ [ton]

Disp $_{m t} \quad$ Disponibilidade de cana $m$ no período $t$ [ton].

\section{Parâmetros da Etapa Industrial}

$\gamma_{t} \quad$ Tempo efetivo de moagem no período $t$ [\%]

Cest ${ }_{p e}$ Capacidade de estocagem do produto $p$ por opção de estoque $e$ no período $t$ [ton ou $\mathrm{m}^{3}$ ]

$h_{p e} \quad$ Custo variável de estocagem do produto $p$ na opção de estoque $e$ [\$/ton ou $\mathrm{m}^{3}$ ]

$h s_{p e} \quad$ Estoque do produto $p$ por opção de estoque $e$ no período de entressafra [ton ou $\mathrm{m}^{3}$ ]

$I_{p e 0} \quad$ Estoque inicial do produto $p$ por opção de estoque $e$ [ton ou $\mathrm{m}^{3}$ ]

$A_{p k t} \quad$ Matriz de rendimento do produto $p$ no processo selecionado $k$ no período $t$ [ton ou $\mathrm{m}^{3}$ ]

$C K_{k t} \quad$ Matriz dos custos industriais. Representa o custo do processo $k$ no período $t$ [\$/ton]

$\mathrm{Ib}_{0} \quad$ Estoque inicial de bagaço [ton]

Fibra $_{m t}$ Quantidade de fibra da cana tipo $m$, no período $t$ [\%]

$\mathrm{Ub}_{t} \quad$ Umidade do bagaço após a moenda, no período $t[\%]$

$\mathrm{Eb}$ Percentual mínimo de estoque de bagaço produzido [\%]

$\mathrm{Epb}$ Estoque de bagaço para passagem de safra [ton]

RC Rendimento médio das caldeiras [ton vapor/ ton bagaço]

RCF Rendimento médio da casa de força [MWh/ ton vapor]

CFVAP Consumo fixo de vapor na moagem [ton de vapor/ton de cana]

$\mathrm{CVAP}_{p}$ Consumo variável de vapor na produção do produto $p$ [ton vapor/ton ou $\mathrm{m}^{3}$ ]

CFE Consumo fixo de energia na moagem [MWh/ ton de cana]

$\mathrm{CVE}_{p} \quad$ Consumo variável de energia na produção do produto $p\left[\mathrm{MWh} /\right.$ ton ou $\left.\mathrm{m}^{3}\right]$

VAPmax Produção diária máxima de vapor [ton/dia]

Egmax Geração diária máxima de energia [MWh/dia]
$V E \quad$ Valor da energia vendida [\$/MWh].

\section{Parâmetros da Etapa Logística}

$D S_{p t} \quad$ Demanda pelo produto $p$ no período $t$ [ton ou $\left.\mathrm{m}^{3}\right]$

$\mathrm{DSP}_{p \text { it }} \quad$ Demanda pelo produto $p$ no período $t$ para o destino $i$ [ton ou $\mathrm{m}^{3}$ ]

$V P_{p t} \quad$ Valor de venda do $p$ no período $t$ [ $\$ /$ ton ou $\left.\$ / \mathrm{m}^{3}\right]$

Disp $_{m t} \quad$ Disponibilidade de cana $m$ no período $t$ [ton]

$I_{p e t} \quad$ Custo de estocagem do produto $p$ na opção de estoque $e$ no período $t$ [ton]

$\mathrm{CAC}_{p i l t}$ Custo do produto $p$ para o destino $i$ usando o transporte $l$ no período $t$ [\$]

DAC $_{p t}$ Demanda do produto $p$ no período $t$ [ton ou $\mathrm{m}^{3}$ ]

DACS $_{p p t}$ Demanda do coproduto $p p$ no período $t$ [ton ou $\mathrm{m}^{3}$ ]

VPS $_{p p t} \quad$ Valor de venda do coproduto $p p$ no período $t$ [\$/ton]

$\mathrm{CS}_{p p t} \quad$ Custo de produção do coproduto $p p$ no período $t\left[\$ /\right.$ ton ou $\left.\$ / \mathrm{m}^{3}\right]$

VVPL $_{p i t}$ Valor de venda do produto $p$ para o destino $i$ no período $t$ [\$ton]

VPS $_{p p t}$ Valor de venda do coproduto $p p$ no período $t$ [\$/ton].

A Tabela 1 contempla alguns parâmetros de entrada (constantes no período de planejamento) e seus valores associados à usina modelada.

Os demais parâmetros, que são muitos, pois são variáveis no tempo, por restrições de espaço, e confidencialidade da Usina estudada, não serão aqui apresentados.

\section{Variáveis de Decisão}

$I_{p e t} \quad$ Quantidade do produto $p$ estocado na opção de estoque $e$ no período $t$ [ton ou $\mathrm{m}^{3}$ ]

$X_{k t} \quad$ Seleção do processo de produção $k$ no período $t$

$M_{m t}^{\prime} \quad$ Quantidade de cana de corte por fonte de

$M_{m t}$ matéria-prima $m$ no período $t$ [ton]

$M_{f t}^{\prime \prime} \quad$ Quantidade de cana transportada por opção

$M_{f t} \quad$ de transporte $f$ no período $t$ [ton]

Disp $_{m t} \quad$ Disponibilidade de matéria-prima $m$ no período $t$ [ton]

$M_{t} \quad$ Quantidade de cana-de-açúcar esmagada no período $t$ [ton]

$M_{k t}^{\prime \prime \prime} \quad$ Quantidade de cana processada no processo

$M_{k t} \quad k$ no período $t$ [ton]

$\mathrm{XAC}_{p \text { ilt }}$ Quantidade do produto $p$ exportada para o destino $i$ usando a opção de transporte $l$ no período $t$ [ton ou $\mathrm{m}^{3}$ ]

$\mathrm{Ib}_{t} \quad$ Estoque de bagaço para geração de energia no período $t$ [ton]

$\mathrm{Mb}_{t} \quad$ Quantidade de bagaço consumido para geração de vapor no período $t$ [ton] 
Tabela 1. Valores adotados para os Parâmetros de Entrada do Modelo MBGP.

\begin{tabular}{|c|c|c|c|c|c|}
\hline Parâmetros & Valores & Parâmetros & Valores & Parâmetros & Valores \\
\hline$M_{\mathrm{t}}^{\min }[$ ton] & 40.000 & $\mathrm{RCF}$ & 0,0765 & $\mathrm{CVE}_{p}$ (Cristal) & 0,018 \\
\hline$M^{\max }{ }_{\mathrm{t}}[$ ton $]$ & 55.000 & CFVAP & 0,235 & $\mathrm{CVE}_{p}(\mathrm{VHP})$ & 0,015 \\
\hline$C T_{f}$ [ton $]$ & 43.000 & $\mathrm{CVAP}_{p}($ Cristal $)$ & 0,0105 & $\mathrm{CVE}_{p}(\mathrm{VVHP})$ & 0,017 \\
\hline $\mathrm{Ib}_{0 t}[$ ton $]$ & 2.000 & $\mathrm{CVAP}_{p}(\mathrm{VHP})$ & 0,0094 & $\mathrm{CVE}_{p}(\mathrm{AEHC})$ & 0,01 \\
\hline $\mathrm{Epb}_{t}[$ ton] & 3.200 & $\mathrm{CVAP}_{p}(\mathrm{VVHP})$ & 0,01 & VAPmax & 4.800 \\
\hline $\mathrm{Eb}[\%]$ & 4 & $\operatorname{CVAP}_{p}($ Cristal $)$ & 1 & Egmax & 240 \\
\hline $\mathrm{RC}$ & 2,3 & CFE & 0,014 & $\mathrm{VE}[\$]$ & 150 \\
\hline
\end{tabular}

VAP $_{t} \quad$ Quantidade de vapor produzido no período $t$ [ton]

$\mathrm{EG}_{t} \quad$ Quantidade de energia produzida no período $t$ [MWh]

$\mathrm{EE}_{t} \quad$ Quantidade de energia exportada no período $t$ [MWh].

\section{Metas associadas aos dez principais objetivos da usina analisada}

Meta do custo total de estocagem [\$];

Meta do custo total da matéria prima (inclui gastos da fase manejo de solo e de corte da cana) [\$];

Meta do custo total do transporte agrícola [\$];

Meta do custo total de produção para os processos [\$];

Meta do custo total de logística para exportação [\$];

Meta de produção do açúcar VHP [ton];

Meta de produção do açúcar VVHP [ton];

Meta de produção do açúcar cristal [ton];

Meta de produção para o etanol [m3];

Meta de produção de energia elétrica [MW];
Número de metas $(\rho)$ que a usina deseja satisfazer plenamente.

\section{Variáveis Auxiliares Específicas para a Função Objetivo}

$y_{\theta} \quad$ Variável binária de controle para a meta $\theta$

$d_{\theta}^{+} \quad$ Variável de desvio associada à obtenção de valor acima do valor alvo da meta $\theta$

$d_{\theta}^{-} \quad$ Variável de desvio associada à obtenção de valor abaixo do valor alvo da meta $\theta$.

\section{Modelo Proposto}

A equação 22 apresenta a função objetivo do modelo MBGP aplicado à usina escolhida como objeto do estudo:

$$
\operatorname{Min} Z=\sum_{\theta \in \Theta}\left(d_{\theta}^{+}+d_{\theta}^{-}\right) \cdot y_{\theta}
$$

\section{Restrições Rígidas (Inflexíveis)}

- Restrição (23) contempla a margem de contribuição global da usina para a safra e entressafra:

$$
\left[\begin{array}{l}
\sum_{p \in P} \sum_{k \in K} \sum_{t \in T} V P_{p t} A_{p k t} M_{k t}^{\prime \prime \prime}+\sum_{p \in P} \sum_{i \in I} \sum_{t \in T} \mathrm{VVPL}_{p i t} D S P_{p i t}+\sum_{p p \in P P} \sum_{t \in T} D A C S_{p p t} \cdot V p s_{p p t}+ \\
+\sum_{t \in T} E E_{t} V E-\sum_{m \in M} \sum_{t \in T} C_{m t} \cdot M_{m t}^{\prime}-\sum_{f \in F} \sum_{t \in T} L_{f t} \cdot M_{f t}^{\prime \prime}-\sum_{k \in K} \sum_{t \in T} C K_{k t} \cdot M_{k t}^{\prime \prime \prime}+ \\
-\sum_{p \in P} \sum_{e \in E} \sum_{t \in T} h_{p e} \cdot I_{p e t}-\sum_{p \in P} \sum_{e \in E} h s_{p e} \cdot I_{p e^{\cdot \prime \prime} T^{\prime \prime}}+ \\
-\sum_{p \in P} \sum_{i \in I} \sum_{l \in L} \sum_{t \in T} \mathrm{CAC}_{p \text { i l }} \cdot D S P_{p \text { i l t }}-\sum_{p p \in P P} \sum_{t \in T} D A C S_{p p} \cdot C s_{p p t}
\end{array}\right] \geq 0
$$

- Restrição (24) considera que apenas um processo de produção deverá ser selecionado em cada período $t$ :

$$
\sum_{k \in K} X_{k t}=1 \quad \forall t \in T
$$

- Restrição (25) calcula a quantidade estocada do produto $\mathrm{p}$ na semana t. Esta restrição permite a transição entre a etapa industrial para a etapa logística:

$$
\begin{aligned}
& \sum_{e \in E} I_{p e t}=I_{0}+\sum_{e \in E} I_{p e t-1}+\sum_{k \in K} A_{p k t} \cdot M_{k t}^{\prime \prime \prime} \\
& -D A C_{p t}-D S_{p i t} \quad \forall p \in P, \forall i \in I, \forall t \in T
\end{aligned}
$$

- Restrições (26-28) identificam a conexão existente entre as variáveis $M_{m t}^{\prime}, M_{f t}^{\prime \prime}, M_{k t}^{\prime \prime \prime}, M_{t}$, em um sistema de único estágio. Estas restrições fazem a transição entre a etapa agrícola e a etapa industrial:

$$
\begin{array}{ll}
\sum_{m \in M} M_{m t}^{\prime}=\sum_{f \in F} M_{f t}^{\prime \prime} & \forall t \in T \\
\sum_{f \in T} M_{f t}^{\prime \prime}=\sum_{k \in K} M_{k t}^{\prime \prime \prime} & \forall t \in T \\
\sum_{k \in K} M_{k t}^{\prime \prime \prime}=M_{t} & \forall t \in T
\end{array}
$$


- Restrição (29) estabelece a quantidade disponível por fonte de matéria-prima $m$ no período $t$ :

$$
\operatorname{Disp}_{m t}=\operatorname{Disp}_{m_{t-1}}-M_{m t-1}^{\prime} \quad \forall m \in M, \forall t \in T \text { (29) }
$$

- Restrição (30) estabelece que toda a cana disponível na safra deve ser inteiramente colhida nesta safra:

$$
\sum_{m \in M} \operatorname{dis} p_{m t}=\sum_{t \in T} m_{t}
$$

- Restrição (31) representa o balanço real da capacidade das moendas no período $t$ :

$$
\begin{aligned}
& \operatorname{Mmin}_{t} \cdot \frac{\varphi_{t}}{100} \cdot \frac{\gamma_{t}}{100} \leq M^{\prime}{ }_{t} \\
& \leq \operatorname{Mmax}_{t} \cdot \frac{\varphi_{t}}{100} \cdot \frac{\gamma_{t}}{100} \quad \forall t \in T
\end{aligned}
$$

- Restrição (32) estabelece a quantidade de cana por tipo $m$ a ser esmagada no período $t$ :

$$
M_{m t}^{\prime} \leq \phi_{t} \cdot M_{t} \quad \forall m \in M, \forall t \in T
$$

- Restrição (33) representa a capacidade de transporte pela frota agrícola $f$ no período $t$ :

$$
M_{f t}^{\prime \prime} \leq \frac{\beta_{f t}}{100} \cdot \frac{\gamma_{t}}{100} . C T_{f} \quad \forall f \in F, \forall t \in T
$$

- Restrição (34) representa a capacidade máxima de cana processada no período $t$.

$$
M_{k t}^{\prime \prime \prime} \leq M^{\max } \cdot X_{k t} \quad \forall k \in K, \forall t \in T
$$

- Restrição (35) representa a capacidade de estocagem do produto $p$ na opção de estoque $e$ no período $t$ :

$$
I_{p e t} \leq \text { Cest }_{p e} \quad \forall p \in P, \forall e \in E, \forall t \in T
$$

\section{Restrições pertinentes à modelagem da cogeração de energia}

Os elementos de cálculo foram os mesmos adotados por Paiva (2009) e Paiva e Morabito (2011).

- Restrição (36) representa o balanço de estoque de bagaço no período $t$ :

$$
I b_{t}=I b_{t-1}+\sum_{m \in M}\left(M_{m t}^{\prime} \frac{\mathrm{Fibra}_{m t}}{1-\mathrm{Ub}_{t}}\right)-\mathrm{Mb}_{t} \quad \forall t \in T \text { (36) }
$$

- Restrição (37) está associada ao estoque de segurança do bagaço no período $t$ :

$$
I b_{t} \geq I b_{t-1}+\sum_{m \in M}\left(M_{m t}^{\prime} \frac{\text { Fibra }_{m t}}{1-\mathrm{Ub}_{t}}\right) . \text { Eb } \forall t \in T \text { (37) }
$$

- Restrição (38) regula o estoque de passagem de bagaço:

$$
I b^{\prime \prime} T^{\prime \prime} \geq \mathrm{EPb} \quad \forall t \in T
$$

- Restrição (39) estabelece a produção de vapor de acordo com a quantidade de bagaço consumido no período $t$ :

$$
\mathrm{Mb}_{t} \cdot \mathrm{RC}=\mathrm{VAP}_{\mathrm{t}} \quad \forall t \in T
$$

- Restrições (40-41) representam o balanço de vapor de alta e baixa pressão de toda a planta industrial no período $t$ :

$$
\begin{aligned}
& \mathrm{VAP}_{\mathrm{t}} \geq \sum_{k \in K} \mathrm{M}_{k t}^{\prime \prime \prime} \cdot \mathrm{CFVAP}+\frac{\mathrm{EG}_{t}}{\mathrm{RCF}} \quad \forall t \in T(40) \\
& \sum_{k \in K} \mathrm{M}_{k t}^{\prime \prime \prime} \cdot \mathrm{CFVAP}+\frac{\mathrm{EG}_{t}}{\mathrm{RCF}} \geq \\
& \sum_{k \in K} \sum_{p \in P} \mathrm{CVAP}_{p} \cdot \mathrm{A}_{p k t} \cdot \mathrm{M}_{k t}^{\prime \prime \prime} \quad \forall t \in T
\end{aligned}
$$

Tabela 2. Valores estabelecidos pela usina para cada meta no Modelo MBGP.

\begin{tabular}{lc}
\hline \multicolumn{1}{c}{ Objetivos e Metas } & Objetivos e Metas \\
\hline Goal $_{1}=\sum_{p \in P} \sum_{e \in E} \sum_{t \in T} h_{p \text { et }} \cdot I_{p e t}=950.000$ & Goal $_{6}=\sum_{p_{1} \in P} \sum_{k \in K} \sum_{t \in T} A_{p k t} \cdot M_{t}=14.500$ \\
ooal $_{2}=\sum_{m \in M} \sum_{t \in T} \mathrm{C}_{m t} \cdot M^{\prime}{ }_{m t}=50.700 .000$ & Goal $_{7}=\sum_{p_{2} \in P} \sum_{k \in K} \sum_{t \in T} A_{p k t} \cdot M_{t}=32.600$ \\
Goal $_{3}=\sum_{f \in F} \sum_{t \in T} l_{f \mathrm{t}} \cdot \mathrm{M}_{f \mathrm{t}}^{\prime \prime}=26.676 .000$ & Goal $_{8}=\sum_{p_{3} \in P} \sum_{k \in K} \sum_{t \in T} A_{p k t} \cdot M_{t}=26.650$ \\
Goal $_{4}=\sum_{k \in K} \sum_{t \in T} C K_{k t} \cdot M_{k t}^{\prime \prime \prime}=9.500 .000$ & Goal $_{9}=\sum_{p_{4} \in P} \sum_{k \in K} \sum_{t \in T} A_{p k t} \cdot M_{t}=85.200$ \\
Goal $_{5}=\sum_{p \in P} \sum_{i \in I} \sum_{l \in L} \sum_{t \in T} C A C_{p i l t} X A C_{p i l t}=1.700 .000$ & Goal $_{10}=\sum_{t \in T} E G_{t}=28.000$
\end{tabular}


- Restrição (42) está associada à quantidade de energia excedente que pode ser consumida em cada período $t$ :

$$
\mathrm{EG}_{t}-\left(\begin{array}{l}
\sum_{k \in K} \mathrm{CFE} \mathrm{M}_{k t}^{\prime \prime \prime}+ \\
\sum_{k \in K} \sum_{p \in P} \mathrm{CVAP}_{p} \cdot \mathrm{A}_{p k t} \cdot \mathrm{M}_{k t}^{\prime \prime \prime}
\end{array}\right)=\mathrm{EE}_{t} \quad \forall t \in T(42)
$$

- Restrições (43-44) estão associadas à capacidade de produção de vapor e energia elétrica no período $t$ :

$$
\begin{array}{ll}
\mathrm{VAP}_{t} \leq \text { VAPMax.nu }_{t} & \forall t \in T \text { (43) } \\
\text { EG }_{t} \leq \text { EGMax. } \varphi_{t} & \forall t \in T \text { (44) }
\end{array}
$$

\section{Restrições Flexíveis (Goal constraints)}

As expressões e valores estabelecidos para as metas, conforme critério já apresentado, estão dispostos na Tabela 2.

- Restrição (45) representa o custo total de estocagem frente à meta estabelecida (Tabela 2):

$$
\left(\sum_{p \in P} \sum_{e \in E} \sum_{t \in T} h_{p e t} . I_{p e t}-950.000\right) \cdot y_{1}=d_{1}^{+}-d_{1}^{-}(45)
$$

- Restrição (46) representa o custo total por fonte de matéria prima frente à meta estabelecida (Tabela 2):

$$
\left(\sum_{f \in F} \sum_{t \in T} l_{f \mathrm{t}} \cdot \mathrm{M}_{f \mathrm{t}}^{\prime \prime}-26.676 .000\right) \cdot y_{2}=d_{2}^{+}-d_{2}^{-}(46)
$$

-Restrição (47) representa o custo total por fonte de matéria prima frente à meta estabelecida (Tabela 2):

$$
\left(\sum_{m \in M} \sum_{t \in T} \mathrm{C}_{m t} \cdot M^{\prime}{ }_{m t}-50.700 .000\right) \cdot y_{3}=d_{3}^{+}-d_{3}^{-}(47)
$$

- Restrição (48) representa o custo total dos processos frente à meta estabelecida (Tabela 2):

$$
\begin{aligned}
&\left(\sum_{k \in K} \sum_{t \in T} C K_{k t} . M_{k t}^{\prime \prime \prime}-9.500 .000\right) \cdot y_{4}=d_{4}^{+}-d_{4}^{-}(48) \quad \begin{array}{r}
\text { - Restrição (56) corresponde às condições de } \\
\text { negatividade e de integralidade das variáveis: }
\end{array} \\
& X_{k t} \in\{0,1\} ; y_{\theta} \in\{0,1\} ; M \geq 0 ; M^{\prime}{ }_{m t} \geq M_{f t}^{\prime \prime} \geq 0 ; M_{k t}^{\prime \prime \prime} \geq 0 ; \\
& \mathrm{XAC}_{p i l t} \geq 0 ; \text { Disp }_{m t} \geq 0 ; I_{p e t} \geq 0 ; \\
& d_{\theta}^{-}, d_{\theta}^{+} \geq 0 ; I b_{t}, M b_{t}, \mathrm{VAP}_{t}, \mathrm{EG}_{t}, \mathrm{EE}_{t} \geq 0 ; \\
& \forall \theta \in \Theta, \forall k \in K, \forall p \in P, \forall M \in M, \forall f \in F, \forall i \in I, \forall t \in T, \forall e \in E, \forall l \in L .
\end{aligned}
$$

\section{Resolução, validação do modelo e discussão dos resultados}

O modelo MBGP para a usina analisada apresentou 1.259 variáveis binárias, 43.894 variáveis não negativas, 9.064 restrições lineares e 2.706 restrições
- Restrição (49) representa o custo total do transporte para exportação frente à meta estabelecida (Tabela 2):

$$
\left(\begin{array}{l}
\sum_{p \in P} \sum_{i \in I} \sum_{l \in L} \sum_{t \in T} C A C_{p i l t} X A C_{p i l t} \\
-1.700 .000
\end{array}\right) \cdot y_{5}=d_{5}^{+}-d_{5}^{-}(49)
$$

- Restrição (50) representa a produção total do açúcar VHP frente à meta estabelecida (Tabela 2):

$$
\left(\sum_{p_{1} \in P} \sum_{k \in K} \sum_{t \in T} A_{p k t} \cdot M_{t}-14.500\right) \cdot y_{6}=d_{6}^{+}-d_{6}^{-}(50)
$$

- Restrição (51) representa a produção total do açúcar VVHP frente à meta estabelecida (Tabela 2):

$$
\left(\sum_{p_{2} \in P} \sum_{k \in K} \sum_{t \in T} A_{p k t} \cdot M_{t}-32.600\right) \cdot y_{7}=d_{7}^{+}-d_{7}^{-}(51)
$$

- Restrição (52) representa a produção total do açúcar Cristal frente à meta estabelecida (Tabela 2):

$$
\left(\sum_{p_{3} \in P} \sum_{k \in K} \sum_{t \in T} A_{p k t} \cdot M_{t}-26.650\right) \cdot y_{8}=d_{8}^{+}-d_{8}^{-}(52)
$$

- Restrição (53) representa a produção total do Etanol frente à meta estabelecida (Tabela 2):

$$
\left(\sum_{p_{4} \in P} \sum_{k \in K} \sum_{t \in T} A_{p k t} \cdot M_{t}-85.200\right) \cdot y_{9}=d_{9}^{+}-d_{9}^{-}(53)
$$

- Restrição (54) representa a geração total de Energia frente à meta estabelecida (Tabela 2):

$$
\left(\sum_{t \in T} E G_{t}-28.000\right) \cdot y_{10}=d_{10}^{+}-d_{10}^{-}
$$

- Restrição (55) estabelece que pelo menos 9 $(\rho=9)$ das 10 metas sejam plenamente atendidas. Isto oferece aos gestores uma flexibilidade para escolher quais são as metas prioritárias para serem atingidas:

$$
\sum_{\theta \in \Theta} y_{\theta}=\rho
$$

- Restrição (56) corresponde às condições de não

não lineares. Para a sua solução, utilizou-se um computador com processador Intel Core $i 7 \mathrm{com}$ tecnologia Max Turbo Frequency, 4MB cache 1,2 GHz até 2,266 GHZ, com 8GB de RAM e sistema Windows 7 profissional 64 bits. O tempo computacional foi de aproximadamente 1 hora e 45 minutos. 
Na Tabela 3, estão resultados que permitem ser efetuada uma comparação com os resultados do modelo e os praticados pela usina, constatando-se a aderência do modelo à realidade.

Analisando-se a Tabela 3, percebe-se que o modelo propõe produzir mais álcool (AEHC) do que açúcar, o que foi coerente com os níveis atuais de produção da usina, que possui contratos que preveem maior demanda pelo álcool, principalmente para exportação.

Observando-se as diferenças entre o que preconiza o modelo, na coluna (a) da Tabela 3, e o que a usina vem realizando, coluna (b) da mesma tabela, tem-se que o modelo apresentou eficiência industrial (ATR da cana/ ATR dos produtos) $0,55 \%$ superior a da usina $(91,3 \%$ contra $90,8 \%)$. Destaca-se que o valor de produção de cada tipo de açúcar foi considerado confidencial pelos diretores da usina e, portanto, não foram divulgados.

$\mathrm{Na}$ coluna (c), constata-se que a margem de contribuição oriunda do modelo foi 8,61\% superior ao resultado obtido pela usina. Com relação à cogeração de energia, o valor proposto pelo modelo ficou muito próximo do valor da usina, o que também mostra uma boa aderência do modelo à realidade.
Aplicou-se o teste de estatístico não paramétrico de Kruskal-Wallis (LU; STEPCHENKOVA, 2012), para averiguar se os resultados do modelo aderiram aos observados na prática (hipótese nula). Em um nível de significância de 5\%, obteve-se $p=0,602$, o que levou à constatação de que a hipótese nula não deveria ser refutada.

$\mathrm{Na}$ Tabela 4, estão os valores das variáveis desvios para cada uma das metas consideradas.

Observando-se a Tabela 4, lembrando que $\mathrm{G}_{6}-\mathrm{G}_{9}$ são as metas de produção, $G_{10}$ é a meta de cogeração de energia e as demais metas $G_{1}-G_{5}$ são as metas de custos para as etapas agrícolas, de produção, de estocagem e de logística, tem-se que:

- A meta $\mathrm{G}_{6}$ atingiu exatamente seu nível de aspiração de 14.500 toneladas;

- A meta $G_{7}$ teve um desvio negativo de (-40) em relação a seu nível de aspiração de 32.600;

- A meta $\mathrm{G}_{8}$ teve um desvio negativo de (-14) sobre seu nível de aspiração de 26.970;

- A meta $\mathrm{G}_{10}$ teve um desvio positivo de $1.176 \mathrm{em}$ relação a seu nível de aspiração de 28.000 (MW);

- Sobre a meta $\mathrm{G}_{9}$ houve um desvio positivo de 38 sobre seu nível de aspiração de 85.200;

Tabela 3. Comparação do modelo MBGP e dados reais da usina.

\begin{tabular}{llccc}
\hline \multicolumn{1}{c}{ Dados } & Modelo MBGP & Usina (2008/2009) & Comparação (\%) \\
\hline & Cristal [ton] & 26.960 & - & - \\
& VHP [ton] & 14.500 & - & - \\
Produtos & 32.560 & - & - \\
& VVHP [ton] & 74.060 & 70.500 & 5,05 \\
& Total produzido de & & 84.660 & 0,64 \\
& açúcares [ton] & 85.238 & 90,8 & 0,55 \\
AEHC [m³] & 91,3 & $12.403 .015(\$)$ & 8,61 \\
Eficiência industrial [\%] & $13.470 .418(\$)$ & $28.000(\mathrm{MW})$ & 4,14 \\
Margem de contribuição [R $\$$ ] & $29.160(\mathrm{MW})$ & & \\
Energia Exportada [R\$] & & &
\end{tabular}

Tabela 4. Valores das variáveis de desvio para cada meta $i$.

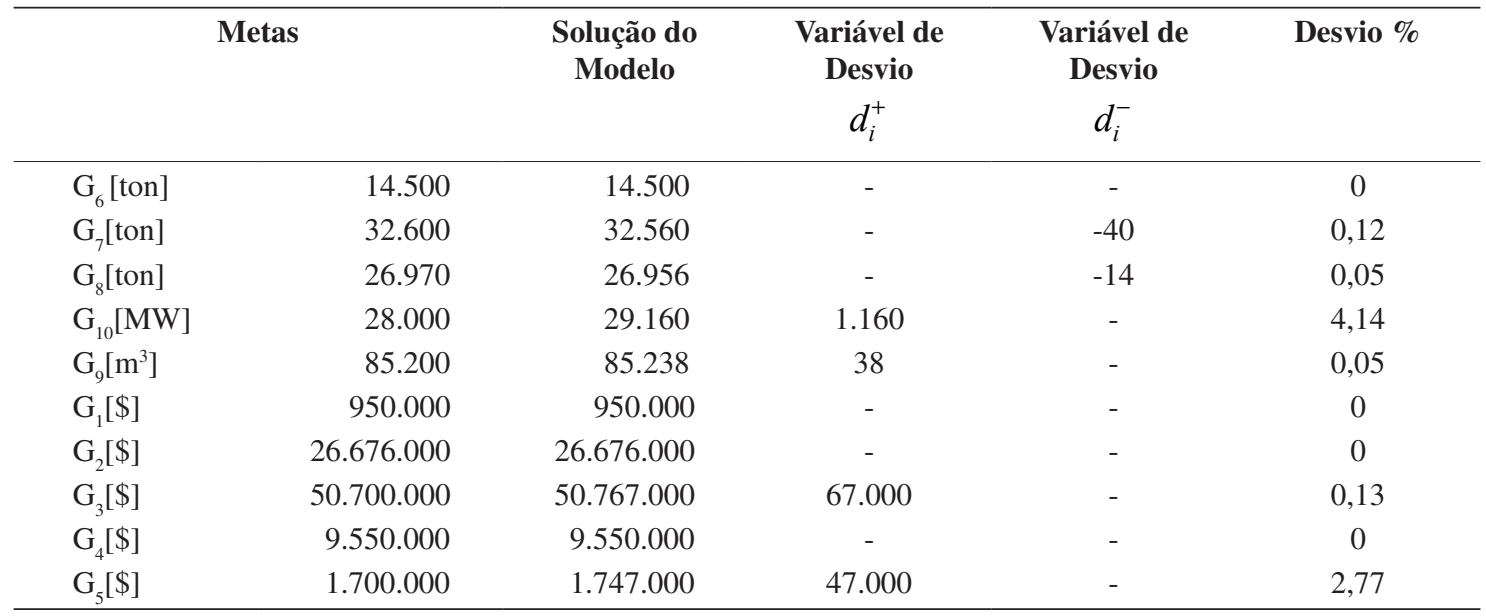


- A meta $\mathrm{G}_{1}$ atingiu exatamente seu nível de aspiração de 950.000;

- A meta $\mathrm{G}_{2}$ atingiu exatamente seu nível de aspiração de 26.676.000;

- A meta $\mathrm{G}_{3}$ teve um desvio positivo de 67.000 sobre o nível de aspiração 50.700.000;

- A meta $\mathrm{G}_{4}$ atingiu exatamente seu nível de aspiração de 9.550.000; e

- $\mathrm{G}_{5}$ teve um desvio positivo de 47.000 sobre o nível de aspiração 1.700.000.

$\mathrm{Na}$ Tabela 4 (6a. coluna), percebe-se que os desvios relativos diante das metas foram muito pequenos, o que também valida a aplicação do modelo MBGP para a usina.

Com relação à inclusão da equação (55) no modelo proposto, na Figura 3, está uma análise de sensibilidade para o tempo de resolução do modelo como função do parâmetro $\rho$, tendo sido adotado no modelo a exigência de que 9 das 10 metas fossem atendidas, visando simular uma situação de maior complexidade computacional.

Cabe mencionar que em todos os cenários simulados (a partir da variação de $\rho$ ) o modelo gerou planos de produção viáveis e eficientes, além de possibilitar um valor maior para a margem de contribuição e, consequentemente, um aumento de competitividade da usina. Por uma questão de espaço, estes resultados foram omitidos neste texto.

Observe-se que a variação do parâmetro $\rho$ permite ao Decisor avaliar o comportamento das múltiplas funções objetivo em relação aos seus valores alvos pré-estabelecidos. Assim, é possível realizar uma análise de sensibilidade para cada cenário, permitindo a exploração de novas estratégias de produção e vendas.

\section{Considerações finais}

Este artigo propôs e validou, para um caso específico, um modelo MBGP para auxiliar nas decisões do planejamento agregado da produção, da logística e da cogeração de energia de uma empresa do setor sucroenergético. De fato, obteve-se a integração das etapas agrícola, industrial e logística em um único modelo MBGP para auxiliar nas decisões de safra e entressafra em um problema real de grande porte.

O modelo permitiu avaliar a eficiência computacional dos Solvers (CONOPT, DICOPT e $C P L E X)$ utilizados para tratar o modelo em função do número de funções objetivo que atingiram o valor alvo fixado para as metas (Figura 3) por meio da variação do parâmetro $\rho$. A variação de $\rho$ também possibilita a geração de cenários, que é uma das contribuições do trabalho, pois auxilia os gestores e tomadores de decisão sobre eventuais mudanças de mercado, e de estratégias empresariais relacionadas à produção e à comercialização.

Deve-se observar que a etapa de implementação do modelo na empresa é de responsabilidade da usina e foge do escopo científico do trabalho, não sendo, portanto discutida aqui. Porém, os ganhos de qualidade no processo decisório, decorrentes da adoção do modelo, ficaram evidentes aos profissionais da usina, notadamente no que se refere ao aumento na margem de contribuição e na eficiência industrial, bem como a possibilidade de simular cenários futuros.

Os profissionais da usina ficaram convencidos de que devem se preocupar com a qualidade das informações dos dados de entrada do modelo e também na escolha criteriosa dos valores para as metas dos objetivos a serem otimizados. Para tanto, o envolvimento da alta gerência deve ser total.

Também é importante comentar que não haveria grandes dificuldades para a empresa incorporar o modelo na obtenção da sua estratégia de planejamento agregado, visto que os pesquisadores tiveram a oportunidade de disseminar os conceitos necessários para o uso e manipulação do modelo, ou seja, a adoção de modelos de Pesquisa Operacional está com o terreno preparado na empresa.

De fato, no desenvolvimento do modelo, o processo interativo ocorrido entre os profissionais da empresa estudada e os pesquisadores, que elaboraram o

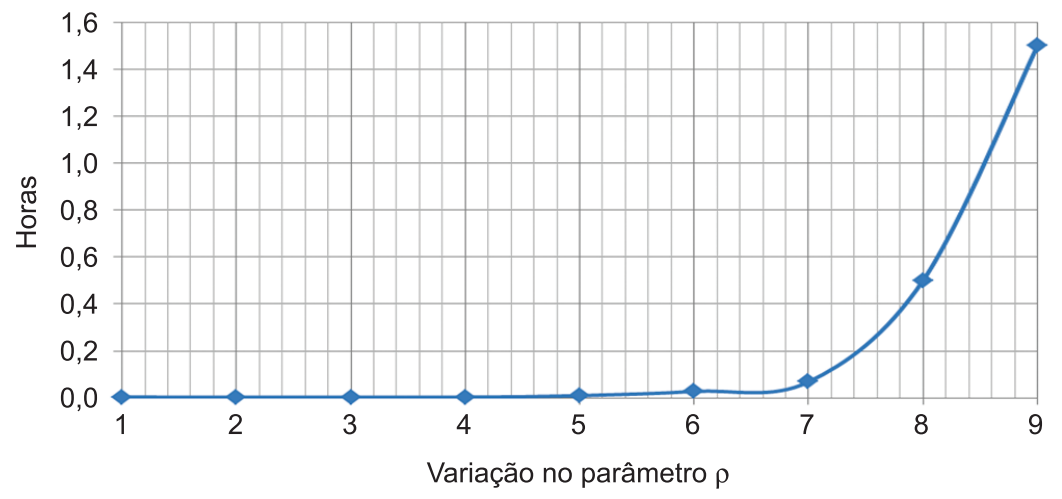

Figura 3. Desempenho computacional dos Solvers com relação ao parâmetro $\rho$. 
modelo, propiciou uma sinergia interessante e permitiu que aspectos relevantes do problema pudessem ser identificados e modelados com maior precisão.

Os resultados da pesquisa aqui relatada são promissores e incentivam sua sequência. Por sugestão e interesse da própria usina, que foi o objeto de estudo deste trabalho, objetivando gerar inovações tecnológicas de uso prático, já está em desenvolvimento pesquisa que aborda a análise dos efeitos das incertezas nos parâmetros de entrada do modelo. Para tanto estão sendo estudadas a Fuzzy Goal Programming e a Multi-Choice Goal Programming (CHANG, 2007, 2008, 2010; YAGHOOBI; TAMIZ, 2007).

Além disto, com finalidade científica, pretende-se:

- Explorar a aplicação de técnicas e conceitos do Projeto de Experimentos (Design of Experiments - DOE), como projetos de experimentos fatoriais e a metodologia de superfície de resposta nos processos industriais, visando reduzir o número de variáveis, e tratar mais adequadamente a não linearidade da matriz de rendimento (MONTGOMERY, 2001);

- Comparar o desempenho computacional do modelo Multi-segment Goal Programming com o modelo de Otimização Estocástica Robusta (LIAO, 2009; MULVEY; RUSZCZYNSKI, 1995; SOYSTER, 1973; YU; LI, 2000; PAIVA, 2009; PAIVA; MORABITO, 2011) em problemas em empresas da área sucroenergética.

\section{Agradecimentos}

Os autores agradecem aos revisores anônimos pelas suas contribuições durante o processo de submissão do artigo e ao CNPq, à CAPES e à FAPEMIG o suporte.

\section{Referências}

AOUNI, B.; KETTANI, O. Goal programming model: A glorious history and a promising future. European Journal of Operational Research, v. 133, n. 2, p. 225-231, 2001. http://dx.doi.org/10.1016/S0377-2217(00)00294-0

AOUNI, B.; MARTEL, J. M.; HASSAINE, A. Fuzzy Goal Programming Model: An Overview of the Current State-of-the Art. Journal of Multi-Criteria Decision Analysis, v. 16, n. 5-6, p. 149-161, 2009. http://dx.doi. org/10.1002/mcda.448

ARAÚJO, S. A.; ARENALES, M. N. Problema de dimensionamento de lotes monoestágio com restrição de capacidade: Modelagem, Método de Resolução e Resultados computacionais. Pesquisa Operacional, v. 20,n. 2, p. 287-306, 2000. http://dx.doi.org/10.1590/ S0101-74382000000200010

BERTRAND, J. W. M.; FRANSOO, J. C. Operations management research methodologies using quantitative modeling. International Journal of Operations and Production Management, v. 22, n. 2, p. 241-264, 2002. http://dx.doi.org/10.1108/01443570210414338
CABALlERO, R.; GÓMEZ, T.; RUIZ, F. Goal programming: Realistic Targets for the Near Future. Journal of Multi-Criteria Decision Analysis, v. 16, n. 3-4, p. 79-110, 2009. http://dx.doi.org/10.1002/ mcda. 442

COCK, J. H.; LUNA, C. A.; PALMA, A. The trade-off between total harvestable production and concentration of the economically useful yield component: cane tonnage and sugar content. Field Crops Research, v. 67, n. 3, p. 257-262, 2000. http://dx.doi.org/10.1016/ S0378-4290(00)00100-3

CUNHA, V.; CAIXETA FILHO, J. V. Gerenciamento da coleta de resíduos urbanos: Estruturação e aplicação de modelo não linear de programação por metas. Gestão \& Produção, v. 9, n. 2, p. 143-161, 2002. http://dx.doi. org/10.1590/S0104-530X2002000200004

CHANG, C-T. An efficient linearization approach for mixed integer problems. European Journal of Operational Research, v. 123, n. 3, p. 652-659, 2000. http://dx.doi. org/10.1016/S0377-2217(99)00106-X

CHANG, C-T. On the mixed binary goal programming problems. Applied Mathematics and Computation, v. 159, n. 3, p. 759-768, 2004. http://dx.doi.org/10.1016/j. amc.2003.11.001

CHANG, C-T. Multi-Choice goal programming. Omega - The International Journal of Management Science, v. 35, n. 4, p. 389-396, 2007.

CHANG, C-T. Revised multi-choice goal programming. Applied Mathematical Modelling, v. 35, n. 4, p. 2587 2595, 2008. http://dx.doi.org/10.1016/j.apm.2007.09.008

CHANG, C-T. Revised multi-choice goal programming for multi-period, multi-stage inventory controlled supply chain model with popup stores in Guerrilla marketing. Applied Mathematical Modelling, v. 34, p. 35863598, 2010. http://dx.doi.org/10.1016/j.apm.2010.03.008

CHARNES, A.; COOPER, W. W. Management model and industrial application of linear programming. New York: Wiley, 1961. v. 1.

CHARNES, A. et al. Chance-constrained Goal Programming model to evaluate response resources for marine pollution disasters. Journal of Environmental Economics and Management, v. 6, n. 3, p. 244-274, 1979. http://dx.doi. org/10.1016/0095-0696(79)90007-X

DEB, K. Multi-Objective Optimization using Evolutionary Algorithms. John Wiley and Sons, 2001.

FERREIRA, D.; MORABITO, R.; RANGEL, S. Solution approaches for the soft drink integrated production lot sizing and scheduling problem. European Journal of Operational Research, v. 169, p. 697-706, 2009. http:// dx.doi.org/10.1016/j.ejor.2008.03.035

GENERAL ALGEBRAIC MODELING SYSTEM - GAMS. Manual do solver DICOPT. GAMS: 2010a. Disponível em: <http://www.gams.com/dd/docs/solvers/dicopt. pdf>. Acesso em: 17 dez. 2010.

GENERAL ALGEBRAIC MODELING SYSTEM - GAMS. Manual do solver CONOPT. GAMS: 2010b. Disponível em: <http://www.gams.com/dd/docs/solvers/conopt. pdf $>$. Acesso em: $17 \mathrm{dez} .2010$.

GENERAL ALGEBRAIC MODELING SYSTEM - GAMS. Manual do solver CPLEX. GAMS: 2010c. Disponível 
em: <http://www.gams.com/dd/docs/solvers/cplex.pdf>. Acesso em: 17 dez. 2010.

GOKCEN, H.; EREL, E. A goal programming approach to mixed-model assembly line balancing problem. International Journal of Production Economics, v. 48, n. 2, p. 177-185, 1997. http://dx.doi.org/10.1016/ S0925-5273(96)00069-2

GRUNOW, M.; GÜNTER, H. O.; WESTINNER, R. Supply optimization for the production of raw sugar. International Journal of Production Economics, v. 110, n. 1-2, p. 110, 224-239, 2007.

HIGGINS, A. J. Scheduling of road vehicles in sugar transport: A case study at an Australian sugar mill. European Journal of Operational Research, v. 170, n. 3, p. 987-1000, 2006. http://dx.doi.org/10.1016/j. ejor.2004.07.055

HIGGINS, A. J. et al. Framework for integrating a complex harvesting and transport system for sugar production. Agricultural Systems, v. 82, n. 2, p. 99-115, 2004. http://dx.doi.org/10.1016/j.agsy.2003.12.004

HIGGINS, A. J.; DAVIES, I. A simulation model for capacity planning in sugar cane transport. Computers and Electronics in Agriculture, v. 47, n. 2, p. 85-102, 2005. http://dx.doi.org/10.1016/j.compag.2004.10.006

IANNONI, A. P.; MORABITO, R. A. Discrete simulation analysis of a logistics supply system. Transportation Research Part E: Logistics and Transportation Review, v. 42, n. 3, p. 191-210, 2006. http://dx.doi.org/10.1016/j. tre.2004.10.002

IGNIZIO, J. P. Goal programming and Extensions. Lexington: Lexington Books, 1976.

JAMALNIA, A.; SOUKHAKIAN, M. A. A hybrid fuzzy goal programming approach with different goal priorities to aggregate production planning. Computers \& Industrial Engineering, v. 56, n. 4, p. 1474-1486, 2009. http:// dx.doi.org/10.1016/j.cie.2008.09.010

JONES, D. F.; TAMIZ, M. Goal programming in the period 1990-2000. In: EHRGOTT, M.; GANDIBLEUX, X. (Eds.). Multicriteria Optimization: State of the Art Annotated Bibliographic Survey. Kluwer Academic Publisher Boston, 2002. chapt. 3.

KAWAMURA, M. S.; RONCONI, D. P.; YOSHIZAKI, H. Optimizing transportation and storage of final products in the sugar and ethanol industry. A case study. International Transactions in Operational Research, v. 13, n. 5, p. 425-439, 2006. http://dx.doi. org/10.1111/j.1475-3995.2006.00556.x

LEUNG, S. C. H.; CHAN, S. S. W. A goal programming model for aggregate production planning with resource utilization constraint. Computers and Industrial Engineering, v. 56, n. 3, p. 138-141, 2009. http://dx.doi. org/10.1016/j.cie.2008.09.017

LIAO, C. N. Formulating the multi-segment goal programming. Computers and Industrial Engineering, v. 56, n. 1, p. 1053-1064, 2009. http://dx.doi.org/10.1016/j. cie. 2008.04 .007

LU, W.; STEPCHENKOVA, S. Ecotourism experiences reported online: Classification of satisfaction attributes. Tourism Management, v. 33, n. 3, p. 702-712, 2012. http://dx.doi.org/10.1016/j.tourman.2011.08.003

MARTEL, J. M.; AOUNI, B. Diverse Imprecise Goal Programming Model Formulations. Journal of Global Optimization, v. 12, n. 2, p. 127-138, 1998. http:// dx.doi.org/10.1023/A:1008206226608
MATHEW, J.; RAJENDRAN, C. Scheduling of maintenance activities in a sugar industry using simulation. Computers in Industry, v. 21, n. 3, p. 331-334, 1993. http://dx.doi. org/10.1016/0166-3615(93)90029-Z

MigUEL, A. C. M. et al. Metodologia de Pesquisa em Engenharia de Produção e Gestão de Operações. 2. ed. Editora Campus, 2012.

MIN, H.; STORBECK, J. On the Origin and Persistence of Misconceptions in Goal Programming. Journal of Operational Research Society, v. 42, n. 3, p. 301-312, 1991.

MILAN, E. L.; FERNANDEZ, S. M.; ARAGONES, L. M. P. Sugar cane transportation in Cuba, a case study. European Journal of Operational Research, v. 174, n. 1, p. 374-386, 2006. http://dx.doi.org/10.1016/j. ejor.2005.01.028

MONTGOMERY, D. C. Design and Analysis of Experiments. 4th ed. New York: Wiley, 2001.

MULVEY, J. M.; RUSZCZYNSKI. A new scenario decomposition method for large-scale stochastic optimization. Operations Research, v. 43, n. 3, p. 477-490, 1995. http://dx.doi.org/10.1287/opre.43.3.477

MUNHOZ, J. R.; MORABITO, R. Um modelo baseado em programação linear e programação de metas para análise de um sistema de produção e distribuição de suco concentrado congelado da laranja. Gestão \& Produção, v. 8, n. 2, p. 139-159, 2001.

MUNHOZ, J. R.; MORABITO, R. Otimização no planejamento agregado de produção em indústrias de processamento de suco concentrado congelado de laranja. Gestão \& Produção, v. 17, n. 3, p. 465-481, 2010. http://dx.doi.org/10.1590/S0104-530X2010000300003

ÖZCAN, U.; TOKLU, B. Multiple-criteria decisionmaking in two-sided assembly line balancing: A goal programming and a fuzzy goal programming models. Computers \& Operations Research, v. 36, n. 6, p. 1955 1965, 2009. http://dx.doi.org/10.1016/j.cor.2008.06.009

PAIVA, R. P. O. Um modelo baseado em seleção de processos e dimensionamento de lotes para o planejamento agregado da produção em usinas de açúcar e álcool. 2006. 182 f. Dissertação (Mestrado em Engenharia de Produção)-Universidade Federal de São Carlos, São Carlos, 2006.

PAIVA, R. P. O; MORABITO, R. Um modelo de otimização para o planejamento agregado da produção em usinas de açúcar e álcool. Gestão \& Produção, v. 14, n. 1, p. 25-41, 2007. http://dx.doi.org/10.1590/ S0104-530X2007000100004

PAIVA, R. P. O. Modelagem do planejamento agregado da produção em usinas cooperadas do setor sucroenergético utilizando programação matemática e otimização robusta. 2009. Tese (Doutorado em Engenharia de Produção)-Universidade Federal de São Carlos, São Carlos, 2009.

PAIVA, R. P. O.; MORABITO, R. An optimization model for the aggregate production planning of a Brazilian sugar and ethanol milling company. Annals Operations Research, v. 169, n. 1, p. 117-130, 2009. http://dx.doi. org/10.1007/s10479-008-0428-9

PAIVA, R. P. O.; MORABITO, R. Programação estocástica robusta aplicada ao planejamento agregado de safra em usinas cooperadas do setor sucroenergético. Gestão \& 
Produção, v. 18, n. 4, p. 719-738, 2011. http://dx.doi. org/10.1590/S0104-530X2011000400004

ROMERO, C. Extended lexicographic goal programming: a unifying approach. Omega, v. 29, n. 1, p. 63-71, 2001. http://dx.doi.org/10.1016/S0305-0483(00)00026-8

ROMERO, C. A general structure of achievement function for a goal programming model. European Journal of Operational Research, v. 153, n. 3, p. 675-686, 2004. http://dx.doi.org/10.1016/S0377-2217(02)00793-2

SAATY, T. L.; SHANG, J. S. Group decision-making: Headcount versus intensity of preference. Socio-Economic Planning Sciences, v. 41, n. 1, p. 22-37, 2007. http:// dx.doi.org/10.1016/j.seps.2005.10.001

SILVA, A. F. Modelagem do planejamento agregado da produção de uma usina sucroalcooleira. 2009. Mestrado (Mestrado em Engenharia de Produção)Universidade Federal de Itajubá, Itajubá, 2009.

SILVA, A. F. et al. A Fuzzy Goal Programming Model for Solving Aggregate Production-Planning Problems under uncertainty: A Case Study in a Brazilian Sugar Mill. In: INFORMS CONFERENCE, 2012, Beijing. Proceedings... Beijing, 2012.

SILVA, A. F. et al. Decisões por múltiplos critérios e objetivos no planejamento agregado da produção e na comercialização e distribuição de uma usina sucroalcooleira. In: SIMPÓSIO DE ENGENHARIA DE PRODUÇÃO, 16., 2009, Bauru. Anais... Bauru, 2009.

SILVA, A. F. et al. timização multiobjetivo fuzzy no planejamento agregado da produção e distribuição em usinas de açúcar e álcool. In: SIMPÓSIO BRASILEIRO DE PESQUISA OPERACIONAL, 42., 2010, Bento Gonçalves. Anais... Bento Gonçalves, 2010.

SILVA, L. M. S. et al. Fitting tapper function to minimize the sum of absolute deviations. Scientia Agricola, v. 63 , n. 5, 2006, p. 460-470. http://dx.doi.org/10.1590/ S0103-90162006000500007

SOYSTER, A. L. Convex programming with set-inclusive constraints and applications to inexact linear programming. Operations Research, v. 21, n. 5, p. 11541157, 1973. http://dx.doi.org/10.1287/opre.21.5.1154

TAMIZ, M.; JONES, D. F.; EL-DARZI, E. A review of Goal Programming and its applications. Annals of Operations Research, v. 58, n. 1, p. 39-53, 1995. http:// dx.doi.org/10.1007/BF02032309

TAMIZ, M.; JONES, D. F.; ROMERO, C. Goal programming for decision making: An overview of the current state-ofthe-art. European Journal of Operational Research, v. 111, n. 3, p. 569-581, 1998. http://dx.doi.org/10.1016/ S0377-2217(97)00317-2

YAGHOOBI, M. A.; TAMIZ, M. A method for solving fuzzy goal programming problems based on MINMAX approach. European Journal of Operational Research, v. 177 , n. 3 , p. 1580-1590, 2007. http://dx.doi. org/10.1016/j.ejor.2005.10.022

USTUN, O. Multi-choice goal programming formulation based on the conic scalarizing function. Applied Mathematical Modelling, v. 36, n. 3, p. 974-988, 2012. http://dx.doi.org/10.1016/j.apm.2011.07.065

YU, C-S.; LI, H. L. A robust optimization model for stochastic logistics problems. International Journal of Production Economics, v. 64, n. 3, p. 385-397, 2000. http://dx.doi.org/10.1016/S0925-5273(99)00074-2

ZIAEE, M.; SADJADI, S. J. Mixed binary integer programming formulations for the flow shop scheduling problems. A case study: ISD projects scheduling. Applied Mathematical Modelling, v. 185, n. 1, p. 218-228, 2007. 